\title{
Technical Facets of a New Methodology to Describe Processes Contemplating Networking of Computer Aided Engineering Methods
}

\author{
Gagan Syal, Nick-Ange Suyam-Welakwe, and Vincent Tixier \\ Daimler AG, Group Research \& Advanced Engineering \\ Wilhelm-Runge-Strasse 11, 89081 Ulm, Germany \\ \{gagan.syal, nick-ange.suyam_welakwe, vincent.tixier\}@daimler.com
}

\begin{abstract}
Computer Aided Engineering (CAE) methods describe behavior of products and simultaneous validate a product design. From scientific and practical viewpoints, these simulation methods have to be executed and synchronized in a process considering their generic interdependencies. For instance while performing car's door digital validation, various simulations have to be performed e.g. stiffness, crash, forming. According to the dependencies, crash simulation needs results of forming simulation as inputs which authenticate a need of CAE process. So far, the CAE process has been accomplished partially and in a manual way, which make engineers unable to validate a complete product in faster and efficient way. A new methodology to develop a CAE network and deriving an explicit product, phase, and priority oriented CAE process from that is described with concept and implementation in this paper. Major objective of this research work is to improve the quality of simulation by developing computer aided network of CAE methods.
\end{abstract}

Keywords: CAE Network, CAE Process, Multiphysics Simulation and Simulation Data Management.

\section{Introduction}

High inspiration to develop the best technical products that satisfy increasing customer requirements on style, quality, comfort, safety, cost and environment protection, is pushing all original equipment manufacturers to work on holistic digital approach from the concept phase to the final phase.

In vehicle development process, the digital validation approach is CAE that enables to simulate almost all the aspects of the product's behaviors in a virtual environment. To validate a product numerous simulations like stiffness analysis, fatigue analysis and fluid analysis are performed. Currently in automotive industries, there is an inefficient interaction among simulations. For holistic design approach a product validation must have to consider dependencies among simulations. For instance, in order to calculate sound pressure level (SPL) around the vehicle occupants' ears, NVH (Noise, Vibration and Harshness) simulation has to be 
performed. In order to calculate the SPL, several inputs are needed, as wind flow evolution, road contact profile, or motor and exhaust behaviors. For a holistic digital approach all these inputs have to be used by NVH in digital form, which is not an optimized process at present. Nowadays, some solutions exist in the area of multidisciplinary simulation; but the factors that define product's holistic behavior and generic dependencies among simulations are not well distinct in digital world [1] [2] [3].

Indeed, the relation between $\mathrm{CAD}, \mathrm{CAE}$ and $\mathrm{CAM}$ in vehicle development process is essential, but this research is concentrated to CAE domain.

\section{State of Art}

\subsection{CAE Process}

Vehicle development process made great stride towards the description of workflow and timeline among interdisciplinary domains as shown in Figure1. The generic dependency between CAD and CAE states that CAD department has to finish the responsibilities and handover to CAE department before freezing date. The workflow between these domains and data transferring is a standardized process. Narrowing to CAE domain, it includes various kinds of simulations like stiffness analysis, Crash Analysis, Aero-dynamics; there is a requisite of standardized data work flow among each other. Currently the data flow is executed manually and partially, which results to error prone and time-consuming process.

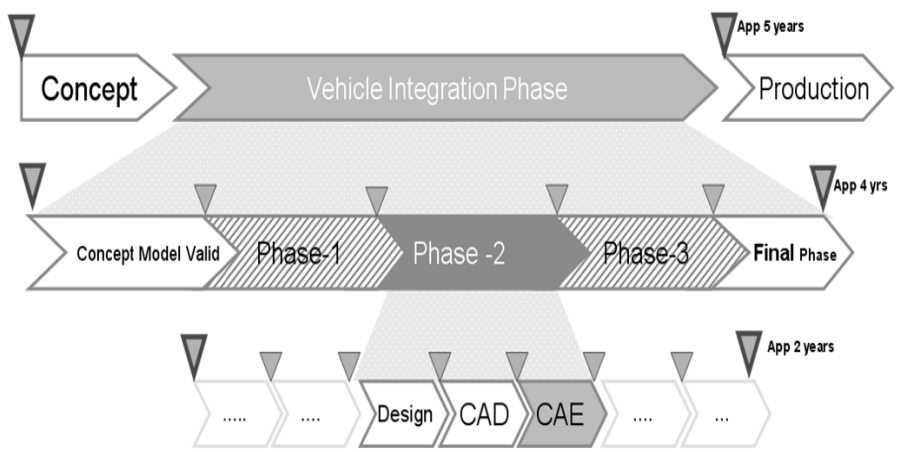

Fig. 1. Vehicle Development Process

Vehicle development process requires a complete integration of CAE process in order to reduce the escalating complexity of simulation data. Presently in most of the industries, CAE process is a parallel process as shown in Figure 2. The simulations are performed independently to each other, even though they are validating a same product. Some processes and solutions help to network simulations but not enough for complete CAE domain. 


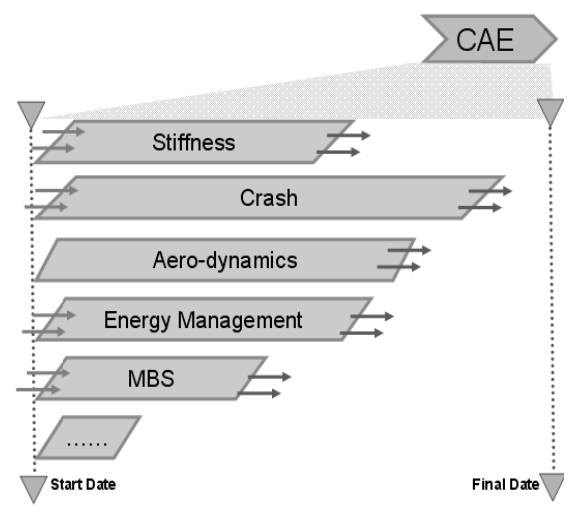

Fig. 2. CAE Phase in Vehicle Development Process

The consequences of deficiency of CAE process are as follows:

- Difficult to co-ordinate the large and versatile CAE structure.

- No controlling on the deadlines.

- No standardized simulation data workflow.

- Manual process results to an error prone and time-Consuming process.

- Unable to validate complete system.

- Dependent on Hardware testing which is a time and money consuming process.

On the contrary, if the workflow among simulations are in the sequential form than it results in extension of development time which is inadequate according to requirements. Time factor plays a major role in describing and optimizing a CAE process. The solutions of these challenges are well described later on. [4][6][15].

\subsection{CAE Networking}

There are different facets of CAE networking. Simulation data management, Multiphysics simulation and co-simulation platforms are common networking methods.

\section{Simulation Data Management:}

Networking of simulation data and results in the data management has not reached the same level of evolution as of product data management systems (PDM systems). Consequently, the results of simulations often fail to fulfill the continuing maturity of product design and development. Feasibility to automate or interpolate various variables is well explained in simulation data management [4] [5] [6] [15].

Multi-physics simulation:

It is a methodology for the design of systems in which strong interactions between disciplines motivates designers to simultaneously manipulate variables in several disciplines. Multidisciplinary tools with numerous interfaces to network FEA, CAD, CFD and control design engineering help to highlight the requirements of digital 
holistic approach; though they cannot currently fulfill the needs for complex automobile parts. [7] [8] [9] [10] [15].

\section{Cosimulation platforms:}

Modern powerful co-simulation platform like ICOS (Independent Co-Simulation Environment) supports the integration of simulation tools from different domains. Modern coupling algorithms ensure that the - in reality existing - interdependencies between different models are depicted. Parallel to this an ITEA-2 project MODELISAR is ensuring a development of Functional Mock-up which is a next generation of the Digital Mock-up to enable the simulation of the vehicle functional behavior. Nevertheless presently these powerful co-simulation platforms cannot fulfill the needs of a digital holistic approach. [2] [11] [15].

\section{A New Methodology}

The innovative methodology is integrated to the existing networking methods and fills the gap, which the existing methods are not able to fill. In this section description of the methodology and technical facets are described.

\subsection{Description of the methodology}

There are two major challenges for building this methodology. First is to create libraries that define the generic dependencies between simulations and between products (in terms of simulation dependencies). These libraries are defines as Generic Relation Matrix (GRM). Second major challenge is to generate automatically a process template according to the generic dependencies.

For a CAE networking, the relation among digital validation methods has to be defined. To characterize the relation following variables has to be defined. The major variables are dependency, mapping, workflow, time as shown in Figure 3.

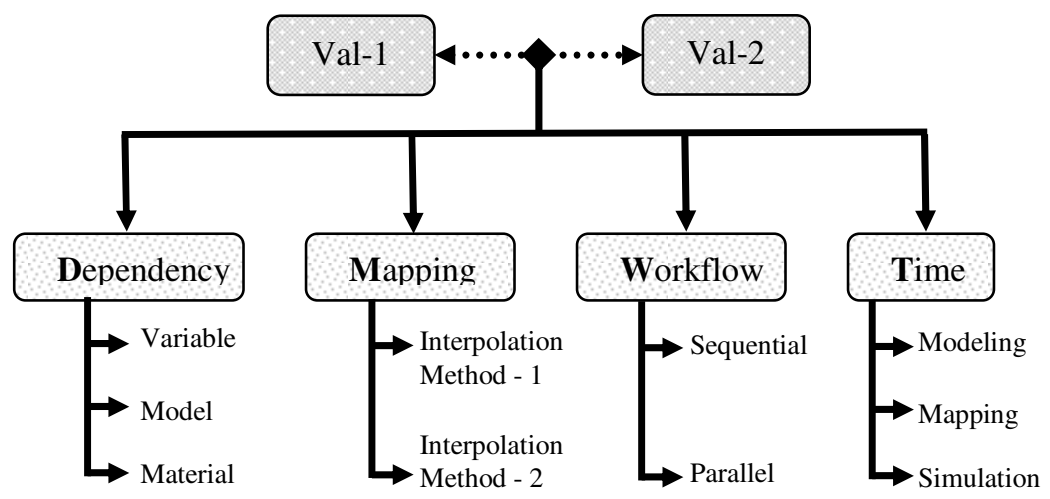

Fig. 3. Generic Validation Relation Factors 
Here is an elaborated example to describe the coupling and generic dependencies between NVH simulation and Aeroacustics simulation. Indeed, NVH (Noise, Vibrations and Harshness) can be caused by several sources, from the vehicle itself as well as from the external environment. One of these sources is the wind which acts as distributed forces. Consequently, NVH simulation requires pressure loads distribution $p(t)$ in time domain in order to be performed.

At present, in several automotive industries, the transient pressure distribution is imported during prototype phase from wind tunnels tests. In order to optimize and fasten the CAE process, the transient data $\mathrm{p}(\mathrm{t})$ can be imported digitally from Aeroacustics simulation (during the CAE phase). This leads to a reduction of dependency on hardware prototypes.

Indeed, Aeroacustics simulation (CFD simulation) is performed using a mesh of the environment, while a mesh of the vehicle is required to perform $\mathrm{NVH}$ simulation (FEA simulation).

The meshes are not matching at the common boundary of both simulations, i.e. the outer surface of the vehicle. Therefore, a digital process called "mapping" must be

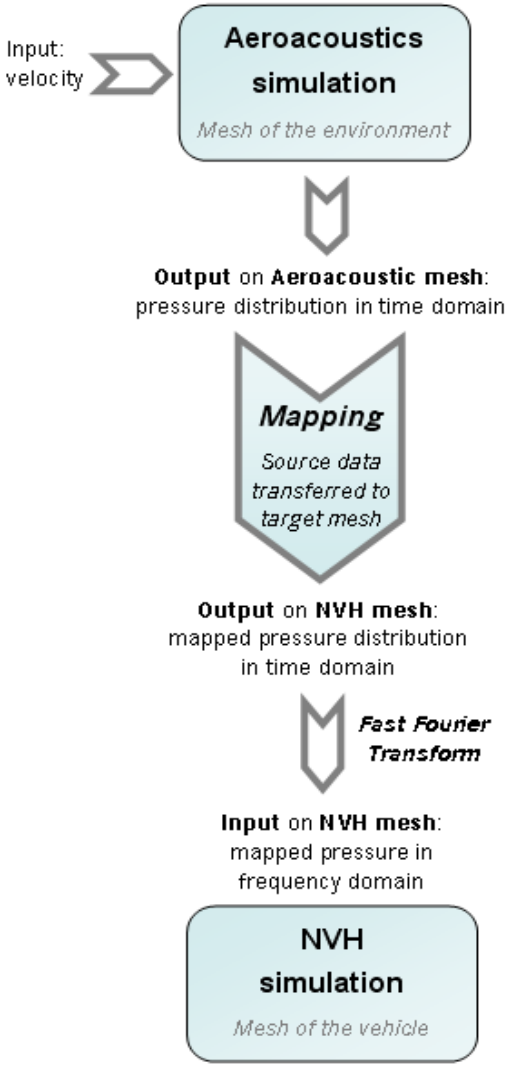

Fig. 4. Simulation Workflow between NVH and Aeroacustics performed. The mapping consists in using a mathematical algorithm (called interpolation) in order to approximate the source model pressure distribution in order to fit with the geometry of the target model. [15][17].

Similarly, the dependency between Forming and Crash simulation is a FE model. The model geometry obtained after forming simulation is used in crash simulation (the initial CAD model can be different in thickness for example). On the other hand, the material properties are modified after forming simulation (compared to initial CAD model which contains classical material properties).

The outcome of such CAE networks increases the quality of simulation as the inputs used to perform NVH or Crash are more in details. Moreover, by CAE networking actual data can be used as compare to prototype results that are performed mostly at the last phase of development process. The CAE network eliminated traditional simplified load cases for which data is imported from prototype testing. Due to the confidentiality and validating the results on various use cases, qualitative results are not mentioned in this paper. 


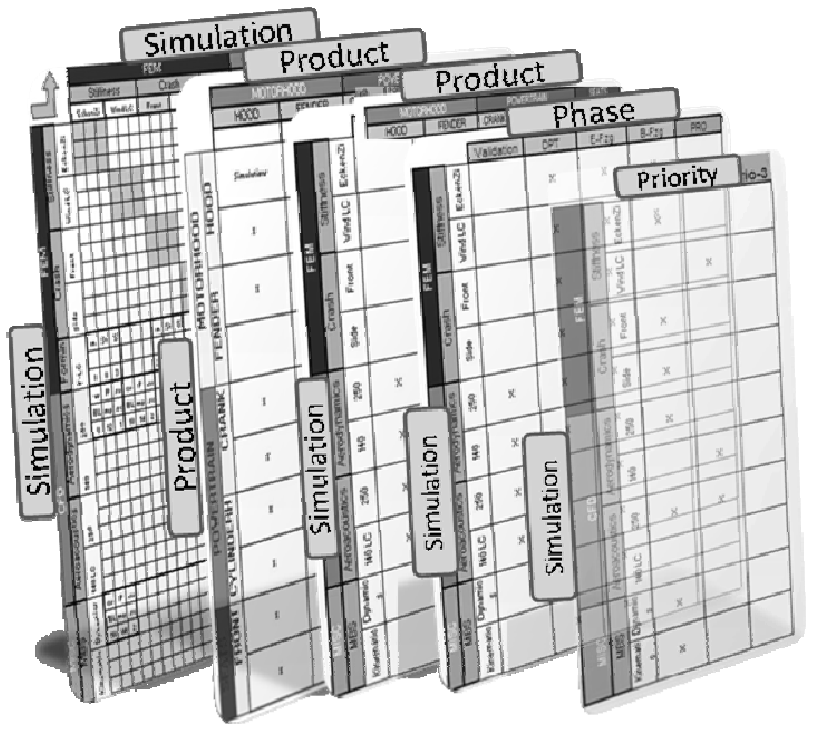

Fig. 5. Generic Relation Matrices (GRM)

Developing of CAE network leads to generic matrices and there are 5 types of relations matrices used in this methodology. Types are:

1. Simulation - Simulation (e.g. Aerodynamics and Stiffness have pressure dependency)

2. Product - Product (e.g. Motor and Motorhood have temperature dependency)

3. Simulation - Product (e.g. Aerodynamics and Exterior Body have decision variable)

4. Simulation - Phase (e.g. Crash - Later Phase also a decision variable)

5. Simulation - Priority

Simulation - Simulation relation matrix is $100 \%$ generic matrix. Simulation -Product and Product - Product relation matrix is generic with respect to automobile industry. The rest of matrices are dependent on companies' requirements.

The most challenging part of this research is the description of a particular process from a GRM shown in Figure 5. An algorithm defines the reduction of GRM volume to a particular product, phase and priority. The first factor which influences the process is the type of product: for example, the interior vehicle's parts do not need to undergo Aerodynamic analysis; as a result, the GRM volume is directly reduced. The next factor is vehicle development phase, e.g. in the early phases of vehicle development, Fatigue analysis plays no role. Similarly, the priority of the digital validation is also important, e.g. in automotive industry. An abstract view of CAE process is shown in Figure 6, the CAE methods are parallel and divided into their activities, dependencies and time line. Different sign modes are used to illustrate the various dependencies and rating simulation quality. 


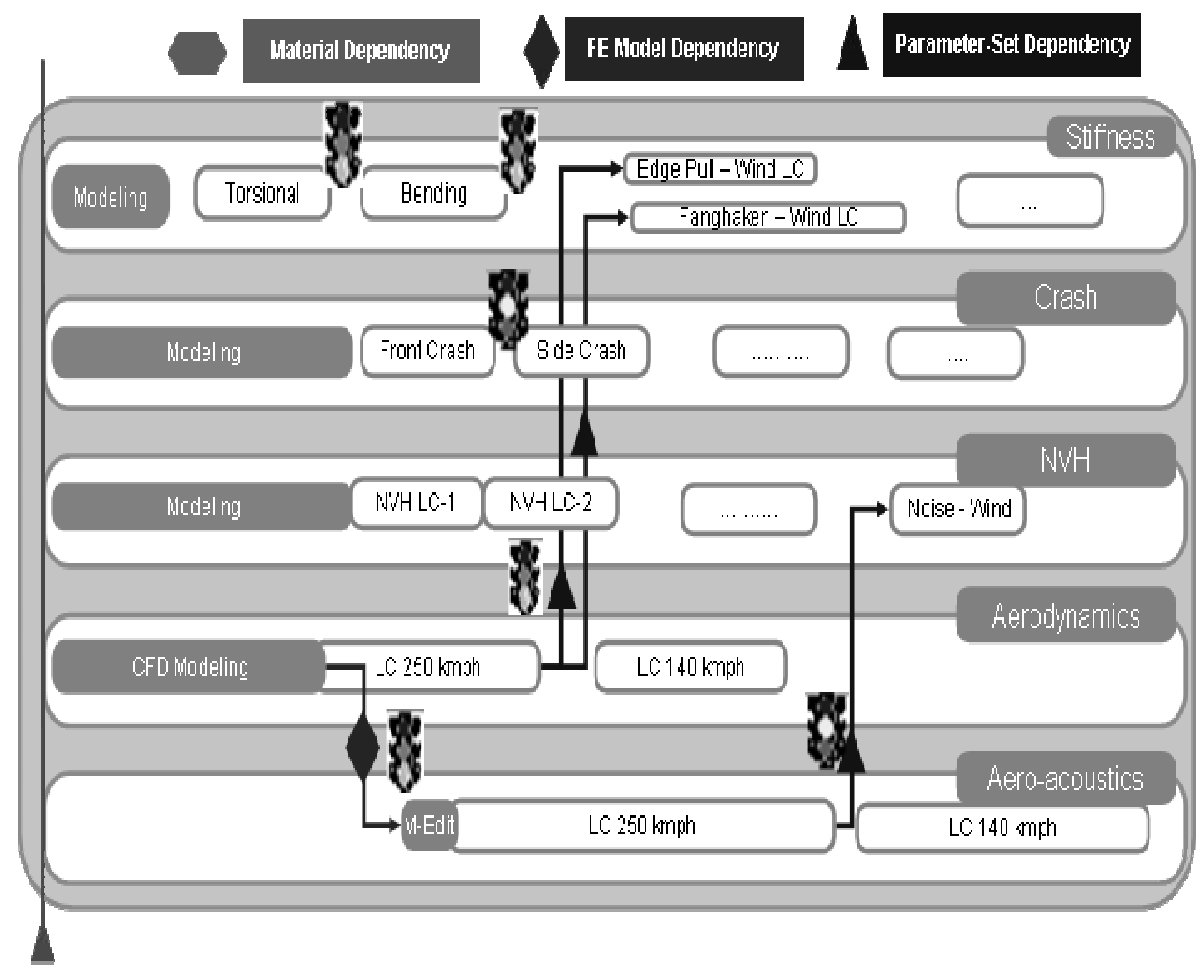

Fig. 6. CAE process derived from the methodology

\subsection{Technical Facets and Implementation of the Methodology}

Technical facets of the methodology are used case diagrams which describe the role of each user, factors to connect the methodology to simulation data management, developing the generic relation matrices and connect the various users in one platform. After continuous discussion with CAE experts and CAE researcher on the new methodology, following common queries towards the conceptual solution figure out:

- How to create the Generic Relation Matrices (GRM)?

- Who will be the creator and user of Generic Relation Matrices (GRM)?

- How to generate the process from the Generic Relation Matrices (GRM)?

- How it is linked to Simulation Data Management and Functional Requirement?

Answers of the queries are portrayed in Figure $7 \mathrm{CAE}$ Networking and Process (CAE-ProNet) which represents whole process of CAE networking. 


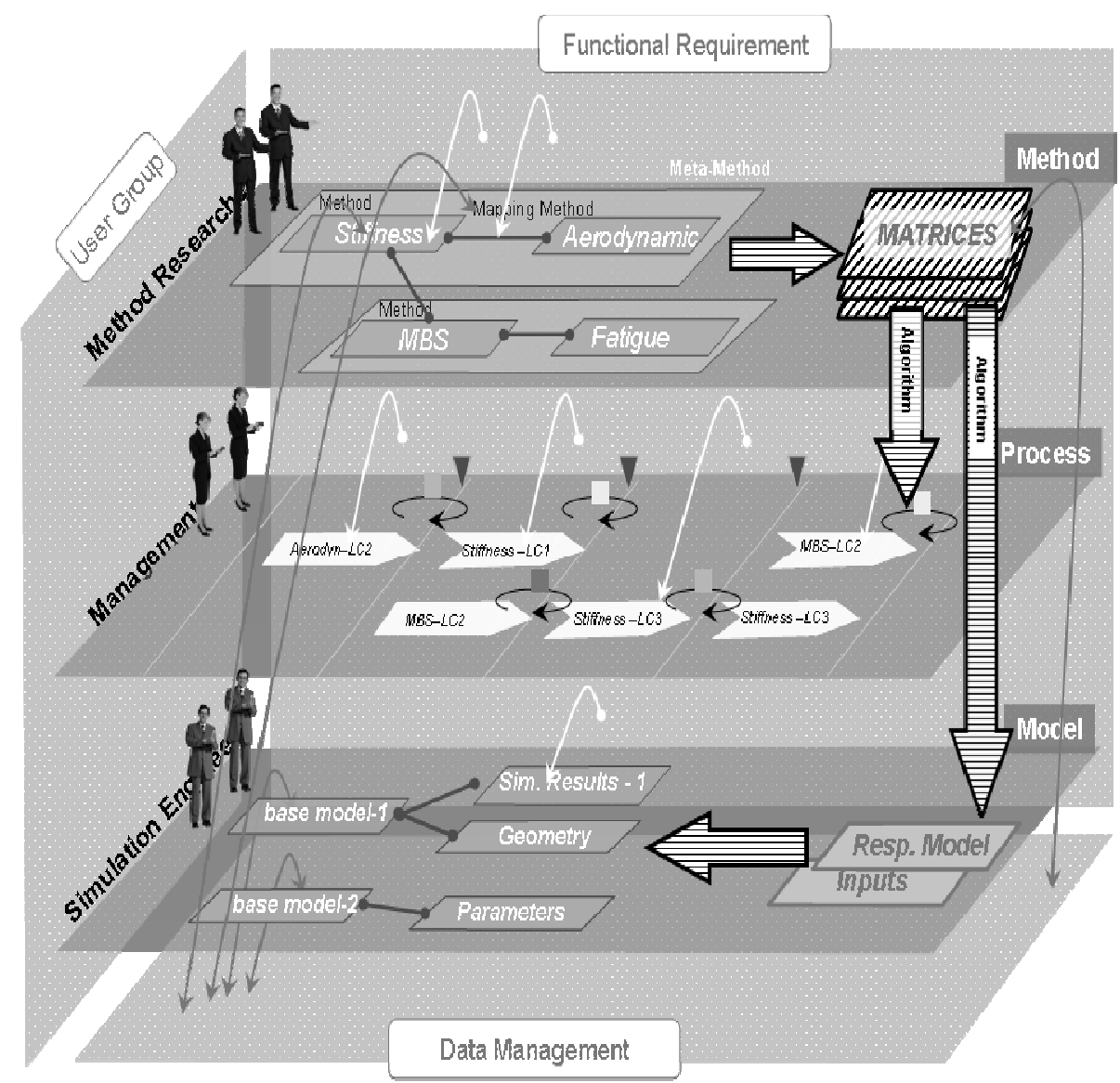

Fig. 7. CAE ProNet Summary Picture

There are three major actors which are responsible for maintaining and developing CAE Network and Process (CAE ProNet). They are method researchers (also known as method engineers), process managers and simulation engineers. The method researchers collect the theoretical information of the simulations, analyze them and define simulation methods and mapping methods for dependent inputs. Validation relation factors are stored in matrices and finally saved in simulation data management. An algorithm is devised to describe the CAE process from generic validation matrices according to the process requirement controlled by process managers. The bottom layer shows the role of the simulation engineers in the CAE process. They receive input data with quality information according to defined CAE process.

As referred from figure 7 that is a huge amount of information being exchanged among described domains and within each domain. The CAE ProNet admin is responsible for the data exchange among given domains. The use case diagram (Figure 8) which is build to develop an application based of activities of each actor are shown below. 


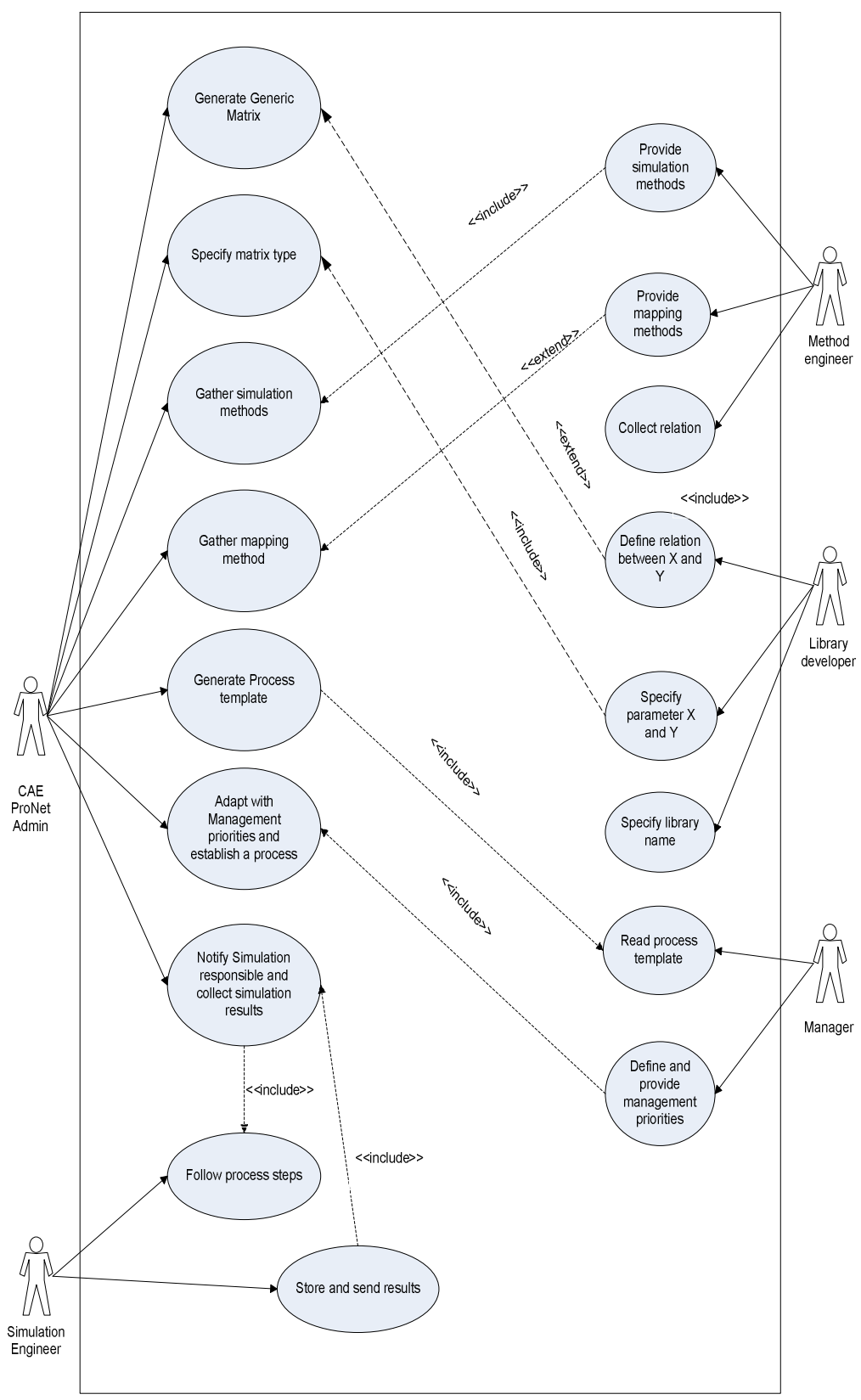

Fig. 8. CAE ProNet - Use case Diagram 


\subsection{Added Value from the New Methodology}

The benefits of the research carried out are as follows:

- Better Simulation Quality:

By means of CAE Networking, inputs received to simulation engineers are in detailed form as compared to simplified, assumption or component based simulation. Thus the results carried out by computational means are better approximation and closer to the real results.

- Better System Organization:

Complexity of simulations and its processes is well managed. The system responsible get an overview about all relevant validations, dependencies, mapping and the process related to the considered system. Effective management decision during the development phases for the dynamic changes.

- Reducing dependencies on Hardware Prototypes:

The methodology results in constructing CAE network which assists dependent simulation to use the digital results of its dependent simulation. Thereby, it reduced the dependency on Hardware prototypes.

- Reducing redundant mapping tool:

One of the aftermaths of CAE network is the reduction of redundant mapping tools.

\section{Related Work}

In the COREPRO project (funded by Uni-Ulm and Daimler AG), fundamental requirements for the IT support of development processes is elaborated. In particular, every subcomponent of the product has related processes that have to be mapped to the overall development process structure and to be synchronized according to the dependencies between the subcomponents. COREPRO is to utilize this information in order to enable the automated coordination data-driven (i.e., product-driven) process structures. The idea is to support the full process life cycle comprising modeling, enactment and change of process structure. The motivation of this project is to link the product dependencies which are similar to this research as linking of CAE dependencies is the focus of this research. [12].

Dissertation by Uwe Gühl on "Design and realization of a modular architecture for a vehicle draft system". It is shown that, especially in the early stage of the car development, there is the possibility to create rapidly car concepts using a modeling tool and to validate these models using simulation tools. In order to address these issues a modular architecture concept is introduced to integrate simulation programs. In a connected relational database the car concept data and configuration data are managed. The project focus was on early stages of car development process and on the function of the product. Other similar projects are objected to CAD-CAE networking [13] [14]. 


\section{Conclusion and Further Approach}

This paper portraits technical facets of a new methodology to develop the CAE network and then driving an explicit product, development phase and priority oriented CAE process. The CAE network by means of generic relation matrices helps every industry to reduce the complexity of managing simulation data. The CAE process helps to improve quality of digital validation and simultaneously reduces the time-tomarket by decreasing the dependencies on hardware prototype. The further approaches are to develop relation matrices between product to product with respect to simulation dependencies and devising an algorithm that describes an explicit process from proposed CAE network.

\section{References}

1. AUTOSIM consortium - Current \& Future Technologies in Automotive Engineering Simulation (CAE), white paper, vol. 6, pp. 08-15 (April 2008)

2. Stippel, H.: Das Virtuelle Fahrzeug Forschungsgesellschaft mbH - CAE-Integration through Co-Simulation, Kompetenzzentrum

3. Schelkle, E.: Up-front CAE Simulation: Just a Catchword or Already a Fact of Modern Automotive Engineering (November 2006)

4. ProSTEP iViP Association - Integration of Simulation and Computation in a PDM Environment (SimPDM). PSI 4, version 2.0, pp. 5-11 (November 2008)

5. Audi, A.G., Gruber, K., Widmann, U., Reicheneder, J., Eberfeld Sidebar, J.: CAE data management. Case Study, 2-6 (2005)

6. Spar Point Research - by Bruce Jenkins Simulation Data Management: Rationalizing the Decision. Best Practice Series - Simulation DM 060620, pp. 1-10 (June 2006)

7. The Pennsylvania State University - Asst. Prof. W.Simpson. Approaches to Multidisciplinary Design Optimization. Lecture Notes. pp. 10-19

8. Palloni, F.: A new tool for multidisciplinary CAE simulation

9. Leuven: FEMTOOLS - FEMtools Programmer's Manual, Dynamic Design Solutions (2004)

10. MpCCI - MpCCI Technical Reference. Fraunhofer SCAI (2007)

11. http://www. itea2.org/projects/step/2

12. Müller, D., Reichert, M., Herbst, J.: A New Paradigm for the Enactment and Dynamic Adaptation of Data-driven Process Structures, research paper (October 2008)

13. Gühl, U.: Design und Realisierungeiner modularen Architektur für ein Fahrzeugentwurfssystem, research paper, pp. 4-7 (September 2001)

14. Hocke, M., Rühle, R., Otter, M.: A Software Environment for Analysis and Design of Multibody Systems

15. Syal, G., Suyam, N.: 8th International workshop on integrated product development: A new methodology to network CAE and describe its process, pp. 1-7 (September 2010)

16. Bercsey, Szelig, Vidovics: 8th International workshop on integrated product development: Time-Estimation of design process based on patterns (September 2010)

17. Tixier, V., Syal, G., Daimler, A.G.: Integration of NVH and Aerodynamics - focusing on the flow of digital solutions, Master Thesis, pp. 55-67 (August 2010) 